PAPER

\section{Exploring negative refraction conditions for quantum cascade semiconductor metamaterials in the terahertz spectral range}

To cite this article: A Danii et al 2016 J. Phys. D: Appl. Phys. 49085105

View the article online for updates and enhancements.
Related content

\begin{tabular}{l} 
- The role of electron-electron scattering in \\
$\frac{\text { gain modulation of a mid-infrared quantum }}{\text { cascade laser in strong magnetic field }}$ \\
Dragan Timotijevi, Jelena Radovanovi and \\
Vitomir Milanovi \\
- Modeling of electron relaxation processes \\
\hline and the optical qain in a magnetic-field \\
\hline A Danisted THz quantum cascade laser \\
- Influence of the active region design on \\
$\frac{\text { output characteristics of GaAs/AIGaAs }}{\text { quantum cascade lasers }}$ \\
J Radovanovi, A Mireti, V Milanovi et al.
\end{tabular}

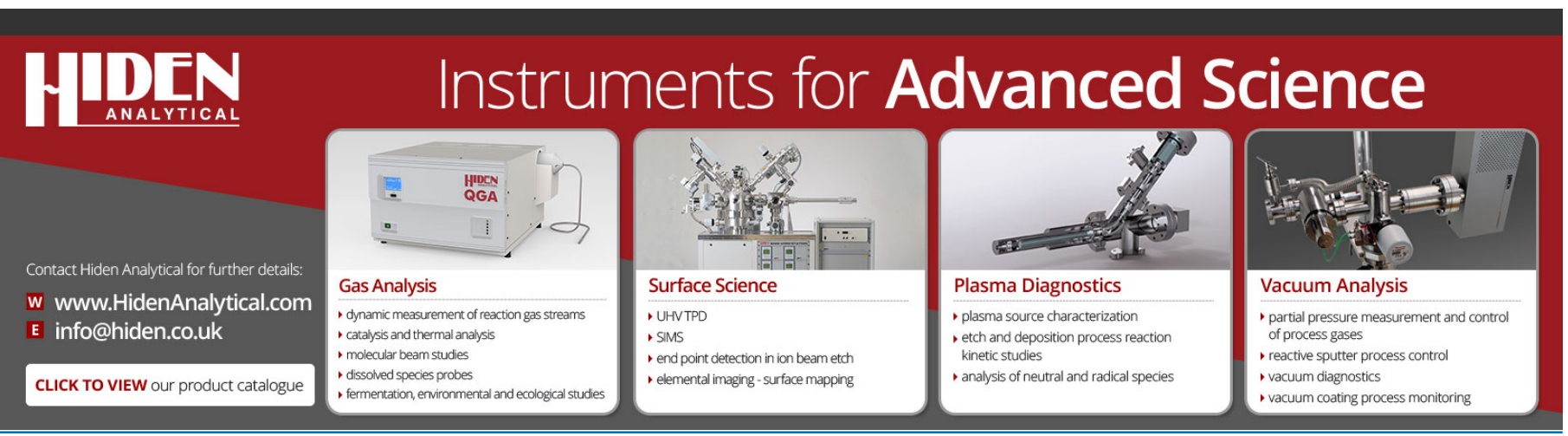




\title{
Exploring negative refraction conditions for quantum cascade semiconductor metamaterials in the terahertz spectral range
}

\author{
A Daničić ${ }^{1}$, J Radovanović ${ }^{2}$, S. Ramović ${ }^{2}$ and V Milanović ${ }^{2}$ \\ ${ }^{1} \mathrm{P}^{*}$ group, Vinča Institute of Nuclear Sciences, POB 522, University of Belgrade, 11001 Belgrade, \\ Serbia \\ ${ }^{2}$ School of Electrical Engineering, University of Belgrade, Bulevar kralja Aleksandra 73, 11120 \\ Belgrade, Serbia \\ E-mail: aleksandar_danicic@vinca.rs
}

Received 16 August 2015, revised 14 December 2015

Accepted for publication 17 December 2015

Published 27 January 2016

\begin{abstract}
In order to avoid losses in metamaterial unit cells at frequencies of interest, caused by metallic inclusions, an active medium design has been proposed. As candidate structures for this active medium, we have chosen quantum cascade lasers because of their high output gain. Here we analyze and compare two quantum cascade structures that emit at $4.6 \mathrm{THz}$ and 3.9 THz, respectively, placed under the influence of a strong magnetic field. We first solve the full system of rate equations for all relevant Landau levels, and obtain the necessary information about carrier distribution among the levels, after which we are able to evaluate the permittivity component along the growth direction of the structure. With these data one can determine the conditions under which negative refraction occurs, and calculate the values of the refractive index of the structure, as well as the range of frequencies at which the structure exhibits negative refraction for a predefined total electron sheet density.
\end{abstract}

Keywords: metamaterials, quantum cascade structures, magnetic field

(Some figures may appear in colour only in the online journal)

\section{Introduction}

During the past decade, a lot of effort has been invested in the development of new artificially structured electromagnetic composites named metamaterials (MTMs), and the investigation of material properties otherwise not found in nature, such as optical magnetism, negative refraction (backward wave propagation), near-zero permittivity, permeability, etc [1-3].

MTMs are comprised of complex constituent elements patterned in periodic arrays, which are significantly smaller than the wavelength of interest so that wave propagation occurs in line with refraction through the effective medium, instead of diffraction, as is the case in photonic crystals [4]. Negative refractive index media were first demonstrated at microwave frequencies [5], which led to the extensive studying of perfect lensing and cloaking phenomena, followed by demonstration of functional MTMs at near-infrared and visible frequencies $[6,7]$, leaving few research attempts concentrating on $\mathrm{THz}$ frequencies. Metallic split-ring resonators have been the most common design for THz 2D planar MTMs; however, some alternative composite right/left-handed metamaterial (CRLM) waveguide designs have been proposed [2, 8, 9] as well. A particular problem in THz MTM realization is caused by the presence of metallic inclusions, which introduce large losses. One way to compensate for the losses is to include an active photonic material in the MTM structure as a source of gain, which is provided via the process of stimulated photon emission [10]. The $\mathrm{THz}$ frequency range is generally very interesting for the investigation of active photonic MTMs, since structures with subwavelength dimensions can 
be readily obtained using modern fabrication techniques, and photonic gain is available through intersubband transitions in THz quantum cascade laser (QCL) [11].

THz QCLs are the longest-wavelength semiconductor laser sources, covering a spectral range of $0.6-5 \mathrm{THz}$. After their first demonstration in 2002 [12], we have witnessed a rapid development of these devices. Now THz QCLs are the only solid-state $\mathrm{THz}$ sources able to deliver average optical power much greater than tens of milliwatts (although cryogenic cooling is still required) essential for applications in $\mathrm{THz}$ imaging, explosive detection and sensing, while their ability to operate in continuous wave regimes makes them suitable for high-resolution $\mathrm{THz}$ spectroscopy $[13,14]$. It could be said that THz QCLs are ideal candidates to fill the 'THz gap'.

In this paper, we have theoretically considered a possible realization of tunable MTMs with structural compositions as in QCLs, capable of delivering high enough values of optical gain required for negative refraction. The analyzed structures comprise very thin GaAs/AlGaAs layers, which are subjected to a strong magnetic field used to enhance the optical gain by manipulating carrier scattering processes, and therefore to control the regime of refraction [15].

\section{Theoretical considerations}

QCL is a low-dimensional semiconductor quantum structure that consists of a series of identical stages. As electron travels through the structure, it (ideally) emits as many photons as the number of periods. The devices should be designed to have two electronic subbands defined as the upper and the lower laser states, electric pumping along the growth direction must be provided, as well as the periodic repetition of active elements, responsible for the light amplification. Due to the specific features of intersubband transitions, the dynamical properties differ from those of interband lasers. Non-radiative intersubband scattering mechanisms are very fast, proceeding on a timescale of a few picoseconds [16]. Although limited by operating temperatures, which are still below the level reachable with Peltier cooling elements, the development of these devices has led to simple active region designs, comprising of only two wells [17], which have the smallest number of quantum-confined subbands involved in electron transport per period. The first QCL demonstration was far more complex, as it was a superlattice-based structure that comprised seven wells [18]. The influence of the external magnetic field on QCL's output properties is of special interest. A lot of research has been made in investigating different scattering mechanisms in magnetic-field-assisted structures, as it may improve QCL performance at longer wavelengths $[15,18]$.

As most of the proposed and so far demonstrated MTM structures include some form of metallic layers (metallic films, wires, spheres, etc), it is expected that those structures exhibit very high optical losses, which could turn out to be detrimental to their performance $[19,20]$. As pointed out above, it is very important to compensate for the losses by using active MTMs, which are able to provide high values of optical gain. Semiconductor quantum structures, in which the layers' widths could be modified (therefore changing the lasing wavelength) while the material composition remains the same, are very important when it comes to choosing the active medium in MTMs. QCLs turn out to be an excellent choice as they may be used to realize substantial optical gain at specific frequencies [21].

The polarization-dependent dipole matrix element that describes intersubband transitions in quantum-well-based nanostructures is responsible for the anisotropy of the optical permittivity tensor. The relative magnetic permeability of a semiconductor-based non-magnetic material is $\mu=1$, while the dielectric permittivity tensor may be written as [20]:

$$
\|\varepsilon\|=\left\|\begin{array}{ccc}
\varepsilon_{\|} & 0 & 0 \\
0 & \varepsilon_{\|} & 0 \\
0 & 0 & \varepsilon_{\perp}
\end{array}\right\|
$$

Here, $\varepsilon_{\|}$is the permittivity component along the quantum well planes, and is equal to $\varepsilon_{\mathrm{b}}$, which denotes the average permittivity of the background material. We neglect the background permittivity dependence on frequency and the doping level (which may introduce changes to the $\varepsilon_{\mathrm{b}}$ values), as we are mostly interested in a qualitative analysis of the effect. Such dependencies may generally be quite important and will be included during further refinements of the model. The intersubband-induced permittivity $\varepsilon_{\perp}$, which describes the interaction along the growth axis, can be represented by the Lorentzian model [22]:

$$
\varepsilon_{\perp}=\varepsilon_{b}+\frac{\mathrm{e}^{2}}{\varepsilon_{0} L \hbar} \sum_{f<i}\left(N_{\mathrm{S}, \mathrm{f}}-N_{\mathrm{S}, \mathrm{i}}\right) \frac{\left|z_{\mathrm{f}, \mathrm{i}}\right|^{2}}{\left(\omega_{\mathrm{i}, \mathrm{f}}-\omega\right)-\mathrm{i} \gamma_{\mathrm{i}, \mathrm{f}}},
$$

where $L$ is the unit cell length in the $z$-direction, $N_{\mathrm{S}, j}$ represents the electron sheet density in the $j$ th state, $\omega_{i, f}$ is the resonant transition frequency between the initial and the final states ( $i$ and $f$, respectively), $\omega$ is the frequency of the input light, $\gamma_{\mathrm{i}, \mathrm{f}}$ stands for the transition linewidth, and $z_{\mathrm{f}, \mathrm{i}}$ denotes the transition dipole matrix element between the final and initial states. As evident from the equation above, the normal component of dielectric permittivity strongly depends on the electron sheet densities in the states of interest. The goal of this work is to meet the criteria for achieving negative refraction, which in the case of anisotropic, single-negative MTM read [23]:

$$
\varepsilon_{\|}>0, \operatorname{Re}\left(\varepsilon_{\perp}\right)<0
$$

It is obvious that in order to obtain negative values of the real part of $\varepsilon_{\perp}$, one has to make sure that high enough population inversion is achieved, in order to cancel out the background term and invert the sign of the real part of $\varepsilon_{\perp}$ in equation (2). Sufficiently high population inversion could potentially be achieved by enhancing carrier injection through the use of high doping densities in the QCL active region. However, high doping densities can in turn lead to complications via space-charge effects and impurity scattering. In this work, we investigate the use of a strong magnetic field as an alternative means of controlling scattering behavior with the aim of avoiding the use of high doping contributions. 

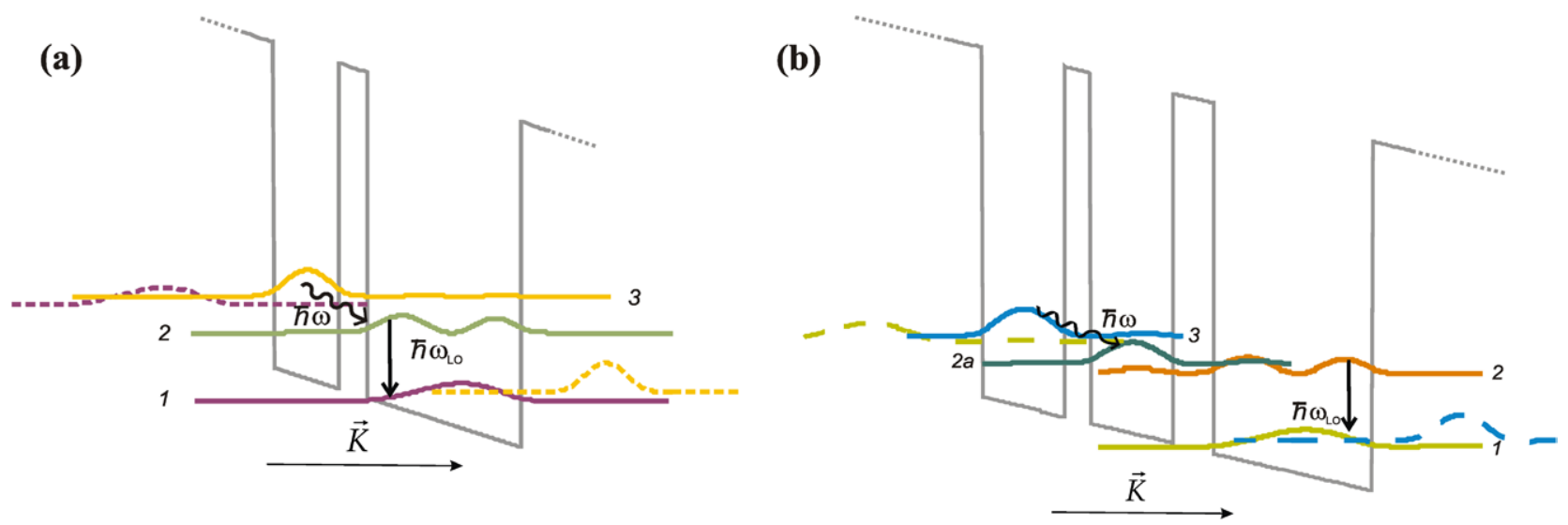

Figure 1. Conduction band profile of a single period of: (a) two-well design lasing at $4.6 \mathrm{THz}$, and (b) three-well design lasing at $3.9 \mathrm{THz}$.

We investigated two QCL active region designs previously reported in the literature $[13,24]$; the first one consists of two quantum wells, while the other design consists of three quantum wells per period, biased by an external electric field. Both structures belong to the $\mathrm{THz}$ part of the spectrum, lasing at $4.6 \mathrm{THz}$ and $3.9 \mathrm{THz}$. The conduction band diagrams of one period of each structure, obtained from our calculations, are presented in figure 1 .

Each period of the structure has three relevant energy states that correspond to the ground state (energy level 1), lower laser state (level 2) and upper laser state (level 3). The laser transition occurs between states 3 and 2, and the corresponding energy difference sets the lasing frequency. The active region is surrounded by collector/emitter barriers, which enable carrier injection from the preceding active region/extraction from the lower subband to the next region [13].

In order to achieve high population inversion $\left(N_{\mathrm{S}, \mathrm{f}}-N_{\mathrm{S}, \mathrm{i}}\right)$, one has to make sure that the lower laser state is being depopulated fast enough. The main mechanism responsible for the depopulation of level 2 is electron-longitudinal optical (LO) phonon scattering. Unlike QCLs operating in the mid-infrared range, where electron-acoustic (AC) phonon scattering may have a contribution in calculating the total relaxation rate, when it comes to THz QCLs this scattering mechanism could be neglected, but interface roughness scattering (IRS) must be taken into account, as there is significant overlap between wavefunctions at the interfaces of adjacent periods. QCL structures that utilize electron-LO phonon scattering as the main depopulation mechanism should be designed in such a manner so that the energy difference $\Delta E_{21}=E_{2}-E_{1}$ approximately equals the LO phonon energy $(\approx 36 \mathrm{meV})$. The advantages of using this particular design are extremely fast depopulation processes, as well as large values of energy separation, which provide intrinsic protection against thermal backfilling [25], as they both allow higher-temperature operation.

The electronic subbands of active regions from figure 1 have free particle-like energy dispersion in the direction parallel to the QW planes; however, in the presence of an external magnetic field $B$ in the direction parallel to the $z$-axis, these continuous subbands split into a series of strictly discrete states called Landau levels, whose energies (including the band nonparabolicity) are given by [15]:

$$
\begin{aligned}
E_{n, l}(B)= & E_{n_{0}}+\left(l+\frac{1}{2}\right) \frac{\hbar e B}{m_{\|, n}\left(E_{n_{0}}\right)}-\frac{1}{8}\left[\left(8 l^{2}+8 l+5\right)\left\langle\alpha_{0}\right\rangle\right. \\
& \left.+\left(l^{2}+l+1\right)\left\langle\beta_{0}\right\rangle\right]\left(\frac{\hbar e B}{m^{*}}\right)^{2}
\end{aligned}
$$

where $E_{n_{0}}$ is the energy of the $n$th state for the zero in-plane wave vector; $m_{\|, n}\left(E_{n_{0}}\right)$ denotes the energy-dependent inplane electron effective mass; $m^{*}=0.0665 m_{0}$, where $m_{0}$ is the free electron mass; $B$ is the value of the magnetic field; $l=0,1,2, \ldots$ is the Landau index; and $\left\langle\alpha_{0}\right\rangle,\left\langle\beta_{0}\right\rangle$ are the nonparabolicity parameters averaged over the $z$-coordinate. The magnetic field clearly affects the Landau levels' energies, so it could be used as a tool to purposely modify the scattering rate between levels of interest, and therefore affect the optical gain, as described in detail in [27].

In order to calculate the refractive index of the structure, one must first find the permittivity, for which the electron distribution over all Landau levels is needed, and it could be calculated by solving the full system of rate equations:

$$
\frac{\partial N_{\mathrm{f}}}{\partial t}=\overline{f_{\mathrm{f}}} \sum_{\mathrm{i} \neq \mathrm{f}} N_{\mathrm{i}} W_{E_{\mathrm{i}} \rightarrow E_{\mathrm{f}}}-N_{\mathrm{f}} \sum_{\mathrm{i} \neq \mathrm{f}} \overline{f_{\mathrm{i}}} W_{E_{\mathrm{f}} \rightarrow E_{\mathrm{i}}}=0 .
$$

Here $i, f$ define the initial and final states, and run through all Landau levels, where all periods are included, and $\bar{f}_{\mathrm{i}}=1-\pi \hbar /(e B) N_{\mathrm{i}}$ relates to the probability of state $i$ being unoccupied. As QCL consists of repetitive elements (periods), the system of rate equations can be simplified as described in [27]. We assume that inside the cascade there is globally linear potential variation, which enables the use of periodic boundary conditions for the carrier surface densities. For each period we assumed an identical set of $N$ Landau levels, with identical electron distribution.

As pointed out, QCL structures could be seen as an anisotropic MTM characterized by a parallel component of the dielectric permittivity $\varepsilon_{\|}$, which has a real and positive value, and a perpendicular component $\varepsilon_{\perp}$, which has a complex value $\varepsilon_{\perp}=\varepsilon_{\perp}^{\prime}+\mathrm{i} \cdot \varepsilon_{\perp}^{\prime \prime}$, and strongly depends on the input frequency. 
Considering the refraction of the incident wave that is passing through a boundary surface between two media (isotropic and anisotropic material), following the Snell law, we calculated the refractive index of the anisotropic medium (QCL):

$n_{\mathrm{QCL}}= \pm \frac{\left|\varepsilon_{\perp}\right|^{2}}{\left|\varepsilon_{\perp}^{\prime}\right| \varepsilon_{\|}} \sqrt{\varepsilon_{\|} \Omega-\varepsilon_{b} \sin ^{2} \theta_{\mathrm{i}}\left\{\frac{\varepsilon_{\|} \varepsilon_{\perp}^{\prime}}{\left|\varepsilon_{\perp}\right|^{2}} \Omega-\left(\frac{\varepsilon_{\|} \varepsilon_{\perp}^{\prime}}{\left|\varepsilon_{\perp}\right|^{2}}\right)^{2}\right\}}$

where $\theta_{\mathrm{i}}$ is the angle of incidence, and $\Omega=(R+\sqrt{R+I}) / 2$, where $R$ and $I$ read:

$$
\begin{gathered}
R=k_{0}^{2} \cdot\left(\frac{1}{\varepsilon_{\|}}-\frac{\varepsilon_{\mathrm{b}} \sin ^{2} \theta_{\mathrm{i}}}{\widetilde{\varepsilon}_{\perp}^{\prime} \varepsilon_{\|}}\right), \\
I=k_{0}^{2} \cdot \frac{\varepsilon_{\mathrm{b}} \sin ^{2} \theta_{\mathrm{i}}}{\widetilde{\varepsilon}_{\perp}^{\prime \prime} \varepsilon_{\|}},
\end{gathered}
$$

where $\widetilde{\varepsilon}_{\perp}^{\prime \prime}=\left|\varepsilon_{\perp}\right|^{2} / \varepsilon_{\perp}^{\prime \prime}$ and $\widetilde{\varepsilon}_{\perp}^{\prime}=\left|\varepsilon_{\perp}\right|^{2} / \varepsilon_{\perp}^{\prime}$, while $k_{0}$ is the wave vector of the incident beam. The sign ' - ' in equation (6) corresponds to the case when $\varepsilon_{\perp}^{\prime}$ has a negative value, while the sign ' + ' stands for the opposite case. The dielectric permittivity of a QCL-based MTM in the direction perpendicular to the structure growth direction is equal to the dielectric permittivity of the bulk material, and reads: $\varepsilon_{\|}=\varepsilon_{\mathrm{b}}=\left(\varepsilon_{\mathrm{b}_{1}} L_{\mathrm{p}_{1}}+\varepsilon_{\mathrm{b}_{2}} L_{\mathrm{p}_{2}}\right) /\left(L_{\mathrm{p}_{1}}+L_{\mathrm{p}_{2}}\right)$, where $L_{\mathrm{p}_{1}}$ and $\varepsilon_{\mathrm{b}_{1}}$ are the width and the bulk dielectric permittivity of the GaAs layer, and $L_{\mathrm{p}_{2}}$ and $\varepsilon_{\mathrm{b}_{2}}$ are the width and the bulk dielectric permittivity of the AlGaAs layer. As $\varepsilon_{b} \approx 13$ for our analysis, the only remaining condition for achieving a negative value of the refractive index is $\varepsilon_{\perp}^{\prime}<0$.

In the case of anisotropic MTMs, the directions of the wavevector and Poynting vector may differ, so one should take into account the refractive index of the wave vector as well when analyzing such a structure. Using the same analysis as previously, we calculate the wave vector refractive index $n_{k}$ :

$$
n_{\mathrm{k}}=\sqrt{\varepsilon_{\|} \Omega_{\mathrm{k}}+\varepsilon_{\mathrm{b}} \sin ^{2} \theta_{\mathrm{i}}\left\{1-\left(\frac{\varepsilon_{\|} \varepsilon_{\perp}^{\prime}}{\left|\varepsilon_{\perp}\right|^{2}}\right)^{2}\right\}}
$$

where $\Omega_{\mathrm{k}}=\left(1+\sqrt{1+\left(I_{\mathrm{k}} / R_{\mathrm{k}}\right)^{2}}\right) / 2$, and

$$
\begin{gathered}
R_{\mathrm{k}}=k_{0}^{2} \cdot\left(\varepsilon_{\|}-\frac{\varepsilon_{\|} \varepsilon_{\mathrm{b}} \sin ^{2} \theta_{\mathrm{i}}}{\left|\varepsilon_{\perp}\right|^{2}} \varepsilon_{\perp}^{\prime}\right), \\
I_{\mathrm{k}}=k_{0}^{2} \cdot \frac{\varepsilon_{\|} \varepsilon_{\mathrm{b}} \sin ^{2} \theta_{\mathrm{i}}}{\left|\varepsilon_{\perp}\right|^{2}} \varepsilon_{\perp}^{\prime \prime} .
\end{gathered}
$$

Unlike in the case of the refractive index of the QC structure, the wave vector refractive index always has a positive value.

\section{Numerical results}

In this paper, two different QCL structures were analyzed, one of them lasing at $4.6 \mathrm{THz}$, with an active region that comprises two QWs, with the other one lasing at $3.9 \mathrm{THz}$, with an active region that has three QWs. Both analyzed structures are realized on a $\mathrm{GaAs} / \mathrm{Al}_{0.15} \mathrm{Ga}_{0.85} \mathrm{As}$ platform. The structure lasing at $4.6 \mathrm{THz}$ is considered to be the simplest structure so far, as having only two wells per period is the minimum required to independently set the energy difference $\Delta E_{21}$, the radiative energy $\Delta E_{32}$, and the radiative matrix element. The layer widths are 56/71/31/167 $\AA$, starting from the emitter towards the collector barrier, and the electric field is set to $17 \mathrm{kV} \mathrm{cm}^{-1}$. By using these data, we calculated the energy difference between subbands 3 and 2 as $20.9 \mathrm{meV}$, while the difference between the lower laser state and the ground state approximately equals the LO phonon energy (36 meV). The structure lasing at 3.9 THz has layer widths 48/85/28/85/42/164 $\AA$, starting from the left barrier, with an electric field of $12.5 \mathrm{kV}$ $\mathrm{cm}^{-1}$. The energy difference between states 3 and 2 reads $15.2 \mathrm{meV}$ according to our calculations, and the energy difference $\Delta E_{21}$ reads $39.6 \mathrm{meV}$. These two designs utilize diagonal transitions which lead to a longer upper-laser-state lifetime and larger population inversion, which are especially important in our case. The numerical results that describe the relaxation rates of these structures are described in detail in [15], where we observed electron-LO phonon scattering and IRS at a temperature of $77 \mathrm{~K}$. As expected, the peaks in scattering rates are found at some values of applied magnetic field, which satisfy the resonance conditions for LO phonon emission from the upper laser state. On the other hand, if there is no Landau level positioned at approximately the LO phonon energy below the state $(3,0)$, this scattering mechanism is forbidden, which consequently increases the upper-laser-state carrier lifetime. It is very important to include other scattering mechanisms in numerical calculations, especially in such cases when a new scattering path may strongly affect the carrier distribution between the laser levels. The total scattering rate of the system can be defined as:

$$
W_{E_{\mathrm{i}} \rightarrow E_{\mathrm{f}}}=W_{E_{\mathrm{i}} \rightarrow E_{\mathrm{f}}}^{\mathrm{LO}}+\sum_{z_{\mathrm{i}}} W_{E_{\mathrm{i}} \rightarrow E_{\mathrm{f}}}^{\mathrm{IRS}}
$$

where $W_{E_{\mathrm{i}} \rightarrow E_{\mathrm{f}}}^{\mathrm{LO}}$ defines the LO phonon scattering rate between the initial and final states, while $W_{E_{\mathrm{i}} \rightarrow E_{\mathrm{f}}}^{\mathrm{IRS}}$ is the IRS rate, and is calculated at every surface $z_{i}$. The modal gain is calculated as in [15], and is presented in figure 2. It was assumed that all the states are broadened, while the broadening of each state is approximated using the Lorentzian distribution function, setting the full width at half maximum parameter to be $\gamma=2 \mathrm{meV}$, which is not dependent on the magnetic field.

Modal gain oscillations with $B$ are very pronounced, as expected, with prominent peaks at $B=7.1 \mathrm{~T}$ and $B=17.5 \mathrm{~T}$ for the first structure, and at $B=3.9 \mathrm{~T}, B=7.6 \mathrm{~T}$ and $B=18.6 \mathrm{~T}$ for the second structure, in line with the absence of LO-phonon resonances. The three-well structure exhibits a dip in modal gain profile around $10.3 \mathrm{~T}$, so in order to use this structure as an effective gain medium, one should apply either a weak magnetic field or a very strong magnetic field (above $17 \mathrm{~T}$ ).

Following equation (2) one can calculate the values of $\varepsilon_{\perp}$. The magnetic field influence on the wave functions is not taken into account, as its effect on the change of the dipole matrix elements can be neglected. For some values of the magnetic field where the peaks appear, it is possible to achieve negative values of the real part of $\varepsilon_{\perp}$ for a fixed total sheet density value. 

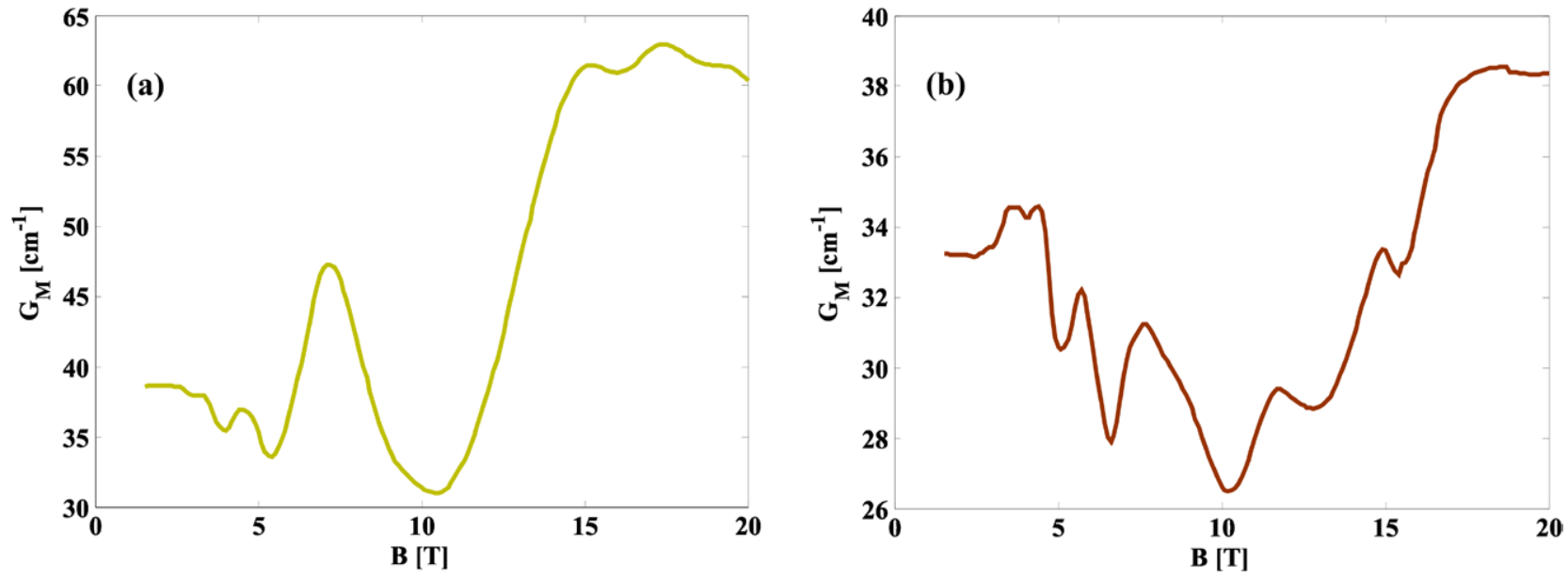

Figure 2. Modal gain as a function of the applied magnetic field: (a) two-well structure lasing at $4.6 \mathrm{THz}$, and (b) three-well structure lasing at $3.9 \mathrm{THz}$.
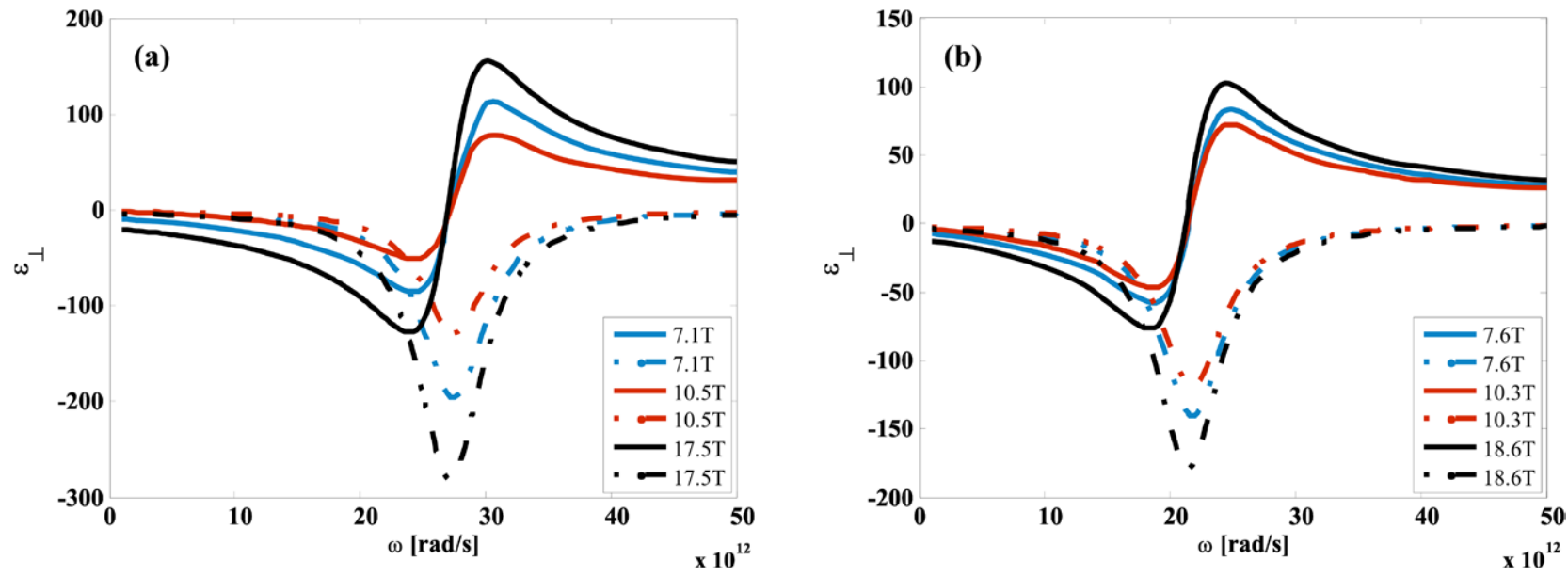

Figure 3. Dependence of the perpendicular component of the dielectric permittivity on the input frequency for: (a) two-well structure lasing at $4.6 \mathrm{THz}$, and (b) three-well structure lasing at 3.9 THz. Solid lines represent the real part, while dotted lines represent the imaginary part of the dielectric permittivity.

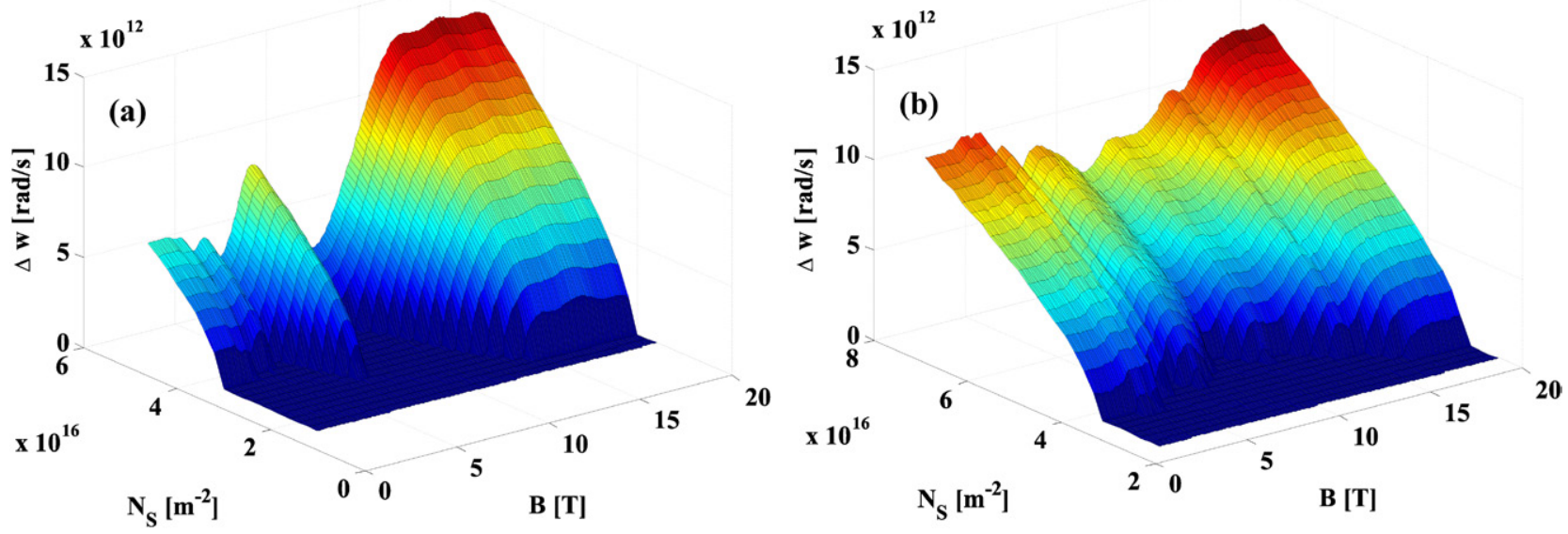

Figure 4. Frequency range at which negative refraction is possible, as it depends on the magnetic field and total sheet density in the case of: (a) two-well structure lasing at $4.6 \mathrm{THz}$, and (b) three-well structure lasing at $3.9 \mathrm{THz}$. 


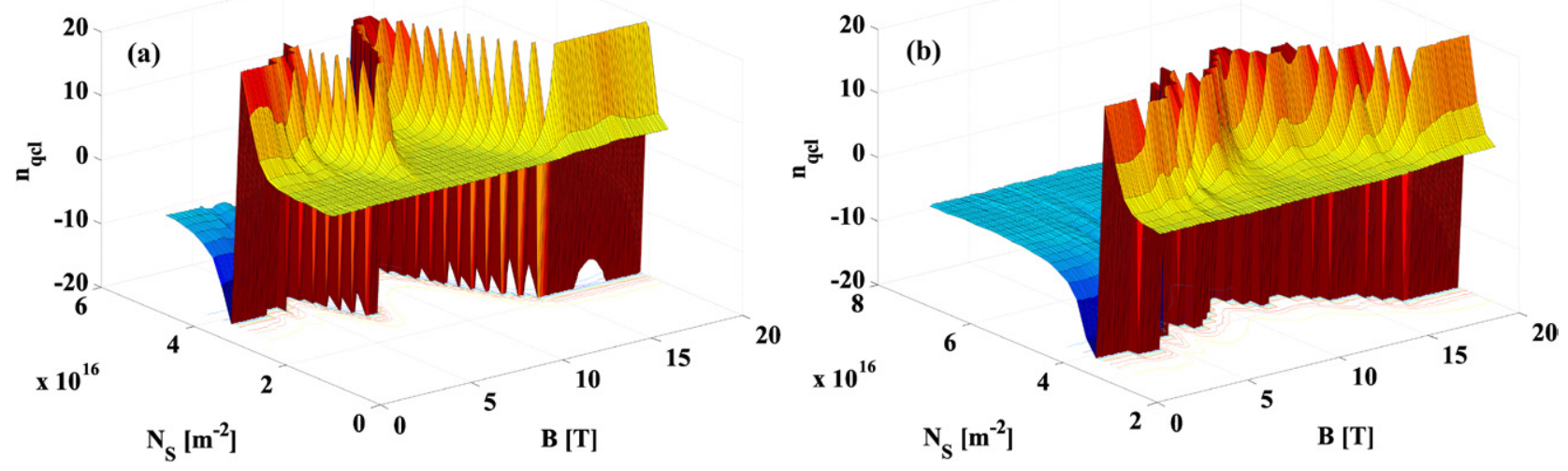

Figure 5. QCL refractive index dependence on magnetic field and total sheet density: (a) two-well structure lasing at $4.6 \mathrm{THz}$, and (b) three-well structure lasing at $3.9 \mathrm{THz}$.
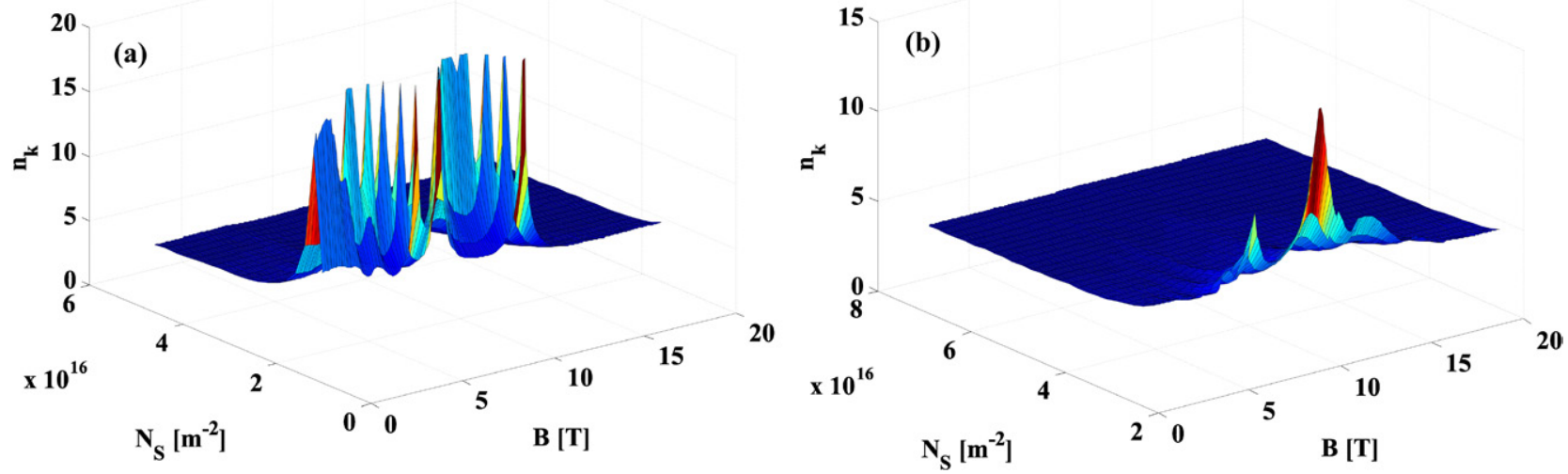

Figure 6. Wave vector refractive index as a function of the total sheet density and magnetic field value for: (a) two-well structure lasing at 4.6 THz, and (b) three-well structure lasing at $3.9 \mathrm{THz}$.

In figure 3 we present how $\varepsilon_{\perp}$ depends on the magnetic field, for the same value of the total sheet density $\left(N_{\mathrm{S}}=2.2 \cdot 10^{17} \mathrm{~m}^{-2}\right)$ for both structures. For each structure it is noticeable that the frequency regions where negative refraction may occur are much wider for magnetic fields which correspond to the peaks in the gain profiles. As expected, for the cases of $B$ where dips in gain profile appear it is more challenging to achieve $\operatorname{Re}\left(\varepsilon_{\perp}\right)<0$; however, in the above example it is still possible due to the rather high values of the total carrier sheet density. Although, in practice, high sheet doping densities will affect the gain (through impurity scattering and contributions to the background permittivity), we have omitted these effects in the present work in order to study the effect of the magnetic confinement independently.

In order to obtain more complete information about the total sheet density required for the onset of negative refraction, we calculated the frequency range with $\operatorname{Re}\left(\varepsilon_{\perp}\right)<0$ as a function of the magnetic field value for numerous total sheet densities (figure 4). Therefore, we can conclude that the minimum value of the total sheet density at which the structure lasing at $4.6 \mathrm{THz}$ exhibits negative refraction equals $2 \cdot 10^{16} \mathrm{~m}^{-2}$ (for the magnetic field value at which the modal gain has the maximum value, $17.5 \mathrm{~T}$ ), and $3.1 \cdot 10^{16} \mathrm{~m}^{-2}$ for the structure lasing at $3.9 \mathrm{THz}$. Although high, these values of the total sheet densities are comparable to those reported in [28] $\left(0.8 \cdot 10^{16} \mathrm{~m}^{-2}\right)$. The total sheet densities for which structures exhibit negative refraction for all values of the applied magnetic field are $4.4 \cdot 10^{16} \mathrm{~m}^{-2}$ and $4.7 \cdot 10^{16} \mathrm{~m}^{-2}$ for the structures lasing at $4.6 \mathrm{THz}$ and $3.9 \mathrm{THz}$, respectively. Although these two structures have very different modal gain profiles, the total sheet densities needed for them to exhibit negative refraction are rather similar.

Following equation (6), and assuming a case of normal incidence, we calculated the values of the refractive indices of the two structures, and the results match the previous conclusion regarding the minimum values of $N_{S}$ at which it is possible to obtain negative values of the refractive index (figure 5). As can be seen from equation (6), we get both negative and positive values of the refractive index, depending on the sign of the real part of the normal component of the dielectric permittivity. If we observe the results in figure 5 , it is obvious that for a fixed value of $n_{\mathrm{QCL}}(x-y$ plane) the shape resembling the structure's modal gain profile is displayed, and for a fixed value of the applied magnetic field ( $y-z$ plane) one can detect an $\varepsilon_{\perp}$-like profile.

Finally, to have a detailed insight into the properties of the structure of interest, one can also calculate the wave vector 
refractive index, following equation (9). The wave vector refractive index as a function of the total sheet density and applied magnetic field is shown in figure 6. In the case of anisotropic structures, such as the ones analyzed in this paper, the wave vector refractive index is different from the refractive index of the structure. The greater the difference, the more anisotropic the structure is.

\section{Conclusion}

The two GaAs/AlGaAs structures considered in this paper emit in the THz part of the spectrum and represent the simplest QCL active region designs, which were experimentally realized and explored [13]. Here, we utilize them to illustrate the possibility of achieving negative refraction, as parts of active-mediumbased MTMs, used to provide large enough gain to yield negative values of the relevant dielectric permittivity component.

Our analysis indicates that an external magnetic field needs to be applied to the structures in order to attain the required values of the gain, as a consequence of the large (positive) background permittivity of GaAs. This magnetic field affects the position of the energy levels and can be used to manipulate the scattering rates and the degree of population inversion between the laser levels, necessary to achieve negative refraction.

Comparing these two structures, one can conclude that not all values of the magnetic field, for which peaks in optical gain occur, can provide large enough values of population inversion in both cases. In some situations, as presented in the numerical results, sufficient population inversion is reached only upon setting quite high values of the total electron sheet density. Nevertheless, the results indicate that negative refraction may be obtained for a variety of magnetic field values, and the required carrier densities and/or values of the magnetic field may be further reduced by the optimization of structural parameters. It would also be worthwhile to investigate other structural designs and material systems with different parameters, in search of a profile capable of exhibiting negative refraction even in the absence of a magnetic field.

\section{Acknowledgments}

This work was supported by the Ministry of Education and Science (Republic of Serbia) (Project III45010) and a NATO SfP Grant, ref. no. 984068.

\section{References}

[1] Veselago V G 1968 Sov. Phys.-Usp. 10 509-14

[2] Schurig D, Mock J, Justice B J, Cummer S A, Pendry J B, Starr A F and Smith D R 2006 Science 264 977-80

[3] Chen H-T, Padilla W J, Zide J M O, Gossard A C, Taylor A J and Averitt R D 2006 Nature 444 576-600

[4] Tavallaee A A, Hon P W C, Mehta K, Itoh T and Williams B S 2010 J. Quantum Electron. 46 1091-8

[5] Shelby R A, Smith D R and Schultz S 2001 Science 292 77-9

[6] Zhou J, Koschny T, Kafesaki M, Economou E N, Pendry J B and Soukoulis C M 2005 Phys. Rev. Lett. 95223902

[7] Grigorenko A N, Geim A K, Gleeson H F, Zhang Y, Firsov A A, Khrushchev I Y and Petrovic J 2005 Nature 438 335-8

[8] Liu Z, Hon P, Tavallaee A A, Itoh T and Williams B S 2012 Appl. Phys. Lett. 100071101

[9] Kildishev A V, Boltasseva A and Shalaev V M 2013 Science 3391232009

[10] Sun L, Yang X and Gao J 2013 Appl. Phys. Lett. 103201109

[11] Radovanović J, Ramović S, Daničić A and Milanović V 2012 Appl Phys. A 109 763-8

[12] Koehler R, Tredicucci A, Beltran F, Beere H E, Linfield E H, Davis A G, Ritchie D A, Iotti R C and Rossi F 2002 Nature 417 156-9

[13] Kumar S 2011 J. Sel. Top. Quantum Electron. 17 38-47

[14] Ferguson B and Zhang X-C 2006 Nat. Mater. 1 26-33

[15] Daničić A, Radovanović J, Indjin D and Ikonic Z 2012 Phys. Scr. T149 014017

[16] Wacker A 2011 Quantum cascade laser: an emerging technology Nonlinear Laser Dynamics (Berlin: Wiley)

[17] Scalari G, Amanti M, Walther C, Terazzi R, Fisher M, Beck M and Faist J 2010 Opt. Express 18 8043-52

[18] Wade A, Fedorov G, Smirnov D, Kumar S, Williams B, Hu Q and Reno J 2009 Nature Photon. 3 41-5

[19] Ginzburg P and Orestein M 2008 J. Appl. Phys. 104063513

[20] Ginzburg P and Orestein M 2008 J. Appl. Phys. 103083105

[21] Ramović S, Radovanović J and Milanović V 2011 J. Appl. Phys. 110123704

[22] Basu P 1997 Theory of Optical Processes in Semiconductors: Bulk and Microstructures (Oxford: Clarendon)

[23] Podolskiy V A and Narimanov E E 2005 Phys. Rev. B 71 201101R

[24] Kumar S, Chan C W I, Hu Q and Reno J L 2009 Appl. Phys. Lett. 95141110

[25] Scalari G, Walther C, Fischer M, Terazzi R, Beere H, Ritchie D and Faist J 2009 Laser Photon. Rev. 3 45-66

[26] Ekenberg U 1989 Phys. Rev. B 40 7714-26

[27] Daničić A, Radovanović J, Milanović V, Indjin D and Ikonić Z 2010 J. Phys. D: Appl. Phys. 43045101

[28] Gabbay A and Brener I 2012 Opt. Express 206584 